\title{
Propagation of Singularities in a Locally Integrable Structure
}

\author{
S. BERHANU
}

\section{Introduction}

In a recent paper [13] Trépreau proved several theorems about the propagation of singularities for $C R$ functions defined on generic $C R$ submanifolds of $\mathbf{C}^{n}$. This work extends some of his results to a general first-order system of PDEs for which the question of holomorphic extendability (more precisely, hypoanalyticity [1]) makes sense.

A few years ago, Hanges and Treves [5] proved that connected elliptic submanifolds of a hypoanalytic manifold $\Omega$ propagate hypoanalyticity of a solution. When $\Omega$ is a $C R$ manifold, elliptic submanifolds coincide with complex submanifolds and "hypoanalyticity of a solution" means holomorphic extendability of a $C R$ distribution. As a corollary of our result, we will derive a microlocal version of the Hanges-Treves theorem; that is, we will get a propagator of microlocal hypoanalyticity in the part of the cotangent space $T^{*} \Omega$ lying above an elliptic submanifold. This corollary will in turn imply the main result of [5].

Another corollary concerns the propagation of microlocal analyticity for solutions of a formally integrable system of real analytic vector fields. It is shown here that the propagation occurs along a Nagano leaf [8] generated by the Hamiltonians of the real and imaginary parts of the vector fields and contained in the characteristic set of the system. We mention that Hanges and Sjöstrand [4] proved such a propagation for solutions of a differential operator of principal type with real analytic coefficients. In the case of systems of analytic vector fields, we believe the approach here is simpler. This paper is also related to the work of Baouendi and Rothschild in [2;3] and that of Tumanov in [14] on wedge extendability in $C R$ manifolds. We mention that our Lemma 4.2 is similar to Lemma 3.1 of [7].

The paper is organized as follows. In Section 1 we will discuss the locally integrable structures we work in and state our main results. Section 2 contains some corollaries to these results. In Section 3 we recall microlocal hypoanalyticity and prove a lemma concerning the wavefront characterization of the FBI transform. In Section 4 we embed our hypoanalytic structure into a $C R$ structure and show that this embedding preserves microlocal 
hypoanalyticity of a solution in certain relevant directions. We also show that this embedding preserves orbits of families of appropriate vector fields both in the base spaces and in the cotangent spaces. Finally, the lemmas of Sections 3 and 4 allow us to use the theorems of Trépreau to prove our theorem.

At this point I would like to thank Professors F. Treves and N. Hanges for several stimulating discussions.

\section{Definitions and Statement of Results}

For the general theory of hypoanalytic structures the reader is referred to [1]. We recall here what we will need. Let $\Omega$ be an open subset of $R^{m+n}$. We suppose, given a $C^{\infty}$ map, that

$$
Z=\left(Z_{1}, \ldots, Z_{m}\right): \Omega \rightarrow \mathbf{C}^{m},
$$

with the differentials $d Z_{1}, \ldots, d Z_{m}$ linearly independent. These differentials generate an $m$-dimensional subbundle of the complex cotangent bundle $C T^{*} \Omega$ which we denote by $T^{\prime} . T^{\prime}$ will be called the structure bundle.

The orthogonal of $T^{\prime}$, denoted by $\mathbf{L}$, for the duality between tangent and cotangent vectors is an $n$-dimensional locally integrable Lie algebra. If $H=$ $\left(H_{1}, \ldots, H_{m}\right)$ is a biholomorphism defined in a neighborhood of $Z(\Omega)$, then $\left\{H_{1}(Z), \ldots, H_{m}(Z)\right\}$ defines the same hypoanalytic structure on $\Omega$.

A distribution $u$ defined in an open subset $V$ of $\Omega$ is called a solution in $V$ if, for any $C^{\infty}$ section $L$ of $\mathbf{L}$ on $V, L u=0$ in the distribution sense in $V$.

Let $X$ be a $C^{\infty}$ submanifold of $\Omega$ of dimension $m$. Denote by $\pi_{X}$ the natural map $\left.T^{*} \Omega\right|_{X} \rightarrow T^{*} X$ and by $\pi_{X}^{\mathrm{C}}$ the analogous map of the complex cotangent bundles. $X$ is called maximally real if $\mathbf{C} T^{*} X=\pi_{X}^{\mathbf{C}}\left(T^{\prime}\right)$. Since a maximally real manifold $X$ is noncharacteristic for $\mathbf{L}$, the trace on $X$ of any solution is well defined.

If $L$ is a smooth section of $\mathbf{L}$, its symbol $\sigma(L)$ vanishes on the bundle $T^{\prime}$. In fact, $T^{\prime}$ is the set of all common zeros of all the functions $\sigma(L)$, with $L$ a smooth section of $\mathbf{L}$. Therefore, Char $\mathbf{L}=T^{*} \Omega \cap T^{\prime}$ is the characteristic set of $\mathbf{L}$. In general, Char $\mathbf{L}$ is not a vector bundle.

A solution $u$ is called hypoanalytic at a point $p$ in $\Omega$ if there is a holomorphic function $\tilde{u}$ defined near $Z(p)$ in $\mathbf{C}^{m}$ such that $u=\tilde{u} \circ Z$ in a neighborhood of $p$. The concept of hypoanalyticity was microlocalized in [1]. In Theorem 1.1 of this section, $W F_{h a}$ will denote the hypoanalytic wavefront set of a solution $u$.

We need to recall briefly from [11] some of the main classes of hypoanalytic structures. The structure defined by $T^{\prime}$ on $\Omega$ is said to be elliptic if Char $\mathbf{L}=0$; it is said to define a $C R$ structure on $\Omega$ if $\mathbf{C} T^{*} \Omega=T^{\prime}+\bar{T}^{\prime}$; it defines a complex structure if it is elliptic and defines a $C R$ structure, in other words, if

$$
\mathrm{C} T^{*} \Omega=T^{\prime} \oplus \bar{T}^{\prime},
$$

where $\oplus$ denotes direct sum. Since the questions we consider are always local, we will work near a central point, say 0 is in $\Omega$. Accordingly, after 
contracting $\Omega$ about 0 and making an affine substitution of the $Z_{j}$ 's and a real local change of coordinates as in [1], we can obtain

$$
\begin{aligned}
& Z_{j}=x_{j}+\sqrt{-1} y_{j}=z_{j} \text { for } j=1, \ldots, r \text { and } \\
& Z_{k}=x_{k}+\sqrt{-1} \Phi_{k}(x, y) \text { for } k=r+1, \ldots, m,
\end{aligned}
$$

with $\Phi_{k}$ real-valued, $\Phi_{k}(0,0)=0$, and $d \Phi_{k}(0,0)=0$ for $k \geq r+1$. The integer $m-r$ is $\operatorname{dim}$ Char $\mathbf{L}$ at 0 . We remark that $T^{\prime}$ is elliptic when $r=m ; T^{\prime}$ is a $C R$ structure when $r=n$; and $T^{\prime}$ is a complex structure when $r=m=n$.

Finally, we need to cite the concepts and results of Sussmann [10] on orbits of families of vector fields. Let $D$ be a set of $C^{\infty}$ vector fields on a $C^{\infty}$ manifold $M$. If $V \in D$, let $\Phi_{V}(t)$ denote the flow of $V$. If $p \in M$, there is a maximal open interval $J(p)$ such that $\Phi_{V}(t) p$ is defined from $J(p)$ into $M$. When we write $\Phi_{V}(t) p$, it will be understood that $t \in J(p)$. Two points $p_{1}$ and $p_{2}$ are said to be $D$-equivalent if there are finitely many vector fields $V_{1}, \ldots, V_{r}$ in $D$ such that

$$
p_{2}=\Phi_{V_{1}}\left(t_{1}\right) \cdots \Phi_{V_{r}}\left(t_{r}\right) p_{1} .
$$

This defines an equivalence relation among the points of $M$. An equivalence class for this relation is called a $D$-orbit. In [10] Sussmann proved that the $D$-orbits are smooth submanifolds of $M$. We will apply this to $D=\operatorname{Re} \mathbf{L}$ and $D=H_{\operatorname{Re} \mathrm{L}}$, where the elements of $\operatorname{Re} \mathrm{L}$ are the real parts of $C^{\infty}$ sections of $\mathbf{L}$ and $H_{\mathrm{Re}} \mathbf{L}$ is the family of Hamilton fields $H_{\sigma(X)}, X \in \operatorname{Re} \mathbf{L}$. The notation $\Theta(D, x)$ will denote the $D$-orbit containing $x$. If $N$ is a submanifold of $\Omega, T_{N}^{*} \Omega$ will denote the conormal of $N$ in $\Omega$.

THEOREM 1.1. Suppose $N$ is a submanifold of $\Omega$ that is a $D$-orbit of $\operatorname{Re} \mathbf{L}$. If $u$ is a solution and $\gamma \in T_{N}^{*} \Omega$, then

$$
\gamma \in W F_{h a} u \Leftrightarrow \mathcal{O}\left(H_{\mathrm{Re}}, \gamma\right) \subseteq W F_{h a} u .
$$

Theorem 1.2. Suppose $\Gamma \subseteq$ Char $\mathbf{L}$ and $\Gamma=\mathcal{O}\left(\gamma, H_{\mathrm{Re}}\right)$ for some $\gamma \in \Gamma$. If $u$ is a solution, then

$$
\gamma \in W F_{h a} u \Leftrightarrow \Gamma \subseteq W F_{h a} u .
$$

\section{Consequences of Theorem 1.1 and Theorem 1.2}

In this section we will first deduce Corollary 2.1 from Theorem 1.1. This corollary will in turn yield Corollary 2.2, which was proved in [5] by Hanges and Treves.

Let $M$ be a submanifold of $\Omega$, and set $T_{M}^{\prime}=\pi_{M}^{C}\left(T^{\prime}\right)$.

Definition 2.1. $\quad M$ is called a hypoanalytic submanifold if it is equipped with a hypoanalytic structure whose structure bundle equals $T_{M}^{\prime}$ and which has the following property:

Given any hypoanalytic function $f$ in an open set $V \subseteq \Omega$, the restriction of $f$ to $M \cap V$ is hypoanalytic. 
If $M$ is a hypoanalytic submanifold of $\Omega$ we call it elliptic, $C R$, complex if these apply to the hypoanalytic structure given to $M$.

Corollary 2.1. Suppose $N$ is an elliptic submanifold of $\Omega$. If $u$ is a solution in $\Omega$ and $\left.\gamma \in \mathrm{Char} \mathbf{L}\right|_{N}$, then

$$
\left.\gamma \in W F_{h a} u \Leftrightarrow \mathcal{O}\left(H_{T N}, \gamma\right) \cap \operatorname{Char} \mathbf{L}\right|_{N} \subseteq W F_{h a} u .
$$

The preceding corollary is a microlocal version of the following corollary, which was the main result in [5].

CoRollary 2.2 (Hanges-Treves [5]). If a solution $u$ is hypoanalytic at a point of a connected elliptic submanifold $M$ of $\Omega$, then $u$ is hypoanalytic at every point of $M$.

Proof of Corollary 2.1. As in [5], we consider three cases.

Case 1: Assume $T^{\prime}$ is a $C R$ structure; that is, assume $r=n$ in (1.1). Then the ellipticity of $N$ implies that it has a complex structure induced by $T^{\prime}$. Assume further that $\operatorname{dim}_{\mathbf{C}} N=n$. Then over $N, \mathbf{L} \oplus \overline{\mathbf{L}}=C T N$. Hence Theorem 1.1 applies to $N$. Let $L_{1}, \ldots, L_{n}$ generate $\mathbf{L}$ (near 0 ). Let $X_{j}=\operatorname{Re} L_{j}$ and $Y_{j}=\operatorname{Im} L_{j}$ for each $j$. Char $\mathbf{L}$ is now a manifold. If $X \in\left\{X_{1}, Y_{1}, \ldots, X_{n}, Y_{n}\right\}$, then $X \in T N$ and therefore $H_{\sigma(X)} \in T\left(\left.C h a r L\right|_{N}\right)$. This fact, together with the equation

$$
\left[H_{\sigma(X)}, H_{\sigma(Y)}\right]=H_{\sigma[X, Y]},
$$

tell us that the family $H_{\mathrm{Re} L}$ foliates Char $\left.\mathbf{L}\right|_{N}=T_{N}^{*} \Omega$ into orbits each of which has dimension $2 n$, and has a basis for its tangent space given by the restrictions of

$$
\left\{H_{\sigma\left(X_{1}\right)}, H_{\sigma\left(Y_{1}\right)}, \ldots, H_{\sigma\left(X_{n}\right)}, H_{\sigma\left(Y_{n}\right)}\right\}
$$

By Theorem 1.1, each of these orbits propagates the singularities of a solution. If $\gamma \in$ Char $\left.\mathbf{L}\right|_{N}$, we clearly have $\mathcal{O}\left(H_{\operatorname{Re} \mathbf{L}}, \gamma\right)=\mathcal{O}\left(H_{T N}, \gamma\right)$.

To deduce Corollary 2.2 in this case, we look at an integral curve for $H_{\sigma\left(X_{j}\right)}$ or $H_{\sigma\left(Y_{k}\right)}$ in an orbit. We use local coordinates $\left(x^{\prime}, x^{\prime \prime}\right) \in \mathbf{R}^{2 n} \times \mathbf{R}^{m-n}$ on $\Omega$ near 0 , which we assume is in $N$, such that $N$ is given by $x^{\prime \prime}=0$. In these coordinates, if

$$
X=\sum_{j=1}^{n+m} a_{j}(x) \frac{\partial}{\partial x_{j}}
$$

is tangent to $N$, then $a_{j}\left(x^{\prime}, 0\right)=0$ for $j>2 n$. Therefore

$$
H_{\sigma(X)}=X-\sum_{t=2 n+1}^{m+n}\left(\sum_{j=2 n+1}^{m+n} \frac{\partial a_{j}}{\partial x_{t}}\left(x^{\prime}, 0\right) \xi_{j}\right) \frac{\partial}{\partial \xi_{t}} \text { over }\left.\operatorname{Char} \mathbf{L}\right|_{N}=T_{N}^{*} \Omega,
$$

where $\xi=\left(\xi_{1}, \ldots, \xi_{m+n}\right)$ is the fiber variable in the cotangent space to $\Omega$. An integral curve $\mathcal{C}(t)$ for $H_{\sigma(X)}$ through $\mathcal{C}(0)=\left.\left(x^{\prime}(0), 0 ; 0, \xi^{\prime \prime}(0)\right) \in \operatorname{Char} \mathbf{L}\right|_{N}$ has the form

$$
\mathcal{C}(t)=\left(x^{\prime}(t), 0 ; 0, \xi^{\prime \prime}(t)\right)
$$


$\xi^{\prime \prime}(t)$ depends linearly on $\xi^{\prime \prime}(0)$ as follows:

$$
\xi^{\prime \prime}(t)=\exp \left(\int_{0}^{t} A(s) d s\right) \xi^{\prime \prime}(0)=B(t) \xi^{\prime \prime}(0),
$$

where $B(t)$ is a matrix depending only on the base projection of the curve $\mathcal{C}$. Since $B(t)$ is invertible, for any fixed $t$ the mapping $\xi^{\prime \prime}(0) \rightarrow B(t) \xi^{\prime \prime}(0)$ from the fiber Char $\mathbf{L}\left(x^{\prime}(0), 0\right)$ to the fiber Char $\mathbf{L}\left(x^{\prime}(t), 0\right)$ is a bijection.

The latter implies Corollary 2.2, since a solution $u$ is hypoanalytic at a point $p$ if and only if its hypoanalytic wavefront set does not intersect Char $\mathbf{L}$ over $p$.

Case 2: $\Omega$ a $C R$ structure as in Case 1, but $\operatorname{dim}_{\mathrm{C}} N=m^{\prime}<n$. Without loss of generality, we may assume that the restrictions of $Z_{1}, \ldots, Z_{m^{\prime}}$ generate the structure bundle on $N$. Since $N$ is a hypoanalytic submanifold, for each $k=m^{\prime}+1, \ldots, m$ there is a holomorphic function $h_{k}$ such that $Z_{k}=$ $h_{k}\left(Z_{1}, \ldots, Z_{m^{\prime}}\right)$ on $N$. We will use the new chart

$$
Z_{1}, \ldots, Z_{m^{\prime}}, Z_{m^{\prime}+1}-h_{m^{\prime}+1}\left(Z_{1}, \ldots, Z_{m^{\prime}}\right), \ldots, Z_{m}-h_{m}\left(Z_{1}, \ldots, Z_{m^{\prime}}\right) .
$$

We also make a real change of coordinates in $\Omega(x, y) \rightarrow(\tilde{x}, \tilde{y})$, where

and

$$
\tilde{x}_{k}= \begin{cases}x_{k} & \text { for } k=1, \ldots, m^{\prime} \\ \operatorname{Re}\left(Z_{k}(x, y)-h_{k}\left(Z_{1}(x, y), \ldots, Z_{m}(x, y)\right)\right) & \text { for } k>m^{\prime}\end{cases}
$$

$$
\tilde{y}_{j}= \begin{cases}y_{j} & \text { for } j=1, \ldots, m^{\prime} \\ \operatorname{Im}\left(Z_{j}(x, y)-h_{j}\left(Z_{1}(x, y), \ldots, Z_{m}(x, y)\right)\right) & \text { for } m^{\prime}+1 \leq j \leq n\end{cases}
$$

After dropping the tildes, we have coordinates $x, y$ and a hypoanalytic chart $Z_{1}, \ldots, Z_{m}$ such that

$$
\begin{gathered}
N=\left\{(x, y): x_{m^{\prime}+1}=\cdots=x_{m}=0, y_{m^{\prime}+1}=\cdots=y_{n}=0\right\} \\
\quad Z_{j}=x_{j}+\sqrt{-1} y_{j} \text { for } j=1, \ldots, n ; \\
Z_{k}=x_{k}+\sqrt{-1} \Phi_{k}(x, y) \text { for } k=n+1, \ldots, m,
\end{gathered}
$$

where $\left.\Phi_{k}\right|_{N}=0$ and $d \Phi_{k}(0,0)=0$.

In these coordinates, a basis $L_{1}, \ldots, L_{n}$ for $\mathbf{L}$ can be chosen so that

$$
L_{j}=\frac{\partial}{\partial \bar{z}_{j}}+\sum_{k=n+1}^{m} a_{k}^{j} \frac{\partial}{\partial x_{k}} \quad \text { when } 1 \leq j \leq n
$$

and $L_{j} \in C T N$ when $1 \leq j \leq m^{\prime}$. In other words,

$$
a_{k}^{j}\left(x^{\prime}, y^{\prime}, 0\right)=0 \text { for } j=1, \ldots, m^{\prime} \text { and } k=n+1, \ldots, m,
$$

Let $\Omega^{\prime}=\left\{(x, y) \in \Omega: y_{m^{\prime}+1}=\cdots=y_{n}=0\right\}$. Equip $\Omega^{\prime}$ with the bundle $\tilde{\mathbf{L}}$ generated by

$$
\tilde{\mathbf{L}}_{j}=\left.L_{j}\right|_{\Omega^{\prime}} \text { for } 1 \leq j \leq m^{\prime} .
$$

Then $\left(\Omega^{\prime}, \tilde{\mathbf{L}}\right)$ is a $C R$ structure containing $N$ as an elliptic submanifold and

$$
\left.\tilde{\mathbf{L}}\right|_{N}=C T N \text {. }
$$


Therefore, by Case 1 , Char $\left.\tilde{\mathbf{L}}\right|_{N}=T_{N}^{*} \Omega^{\prime}$ is a union of orbits along each of which the singularities of solutions in the structure $\left(\Omega^{\prime}, \tilde{\mathbf{L}}\right)$ propagate. From (2.1), we get

$$
\left.\operatorname{Char} \mathbf{L}\right|_{N} \subseteq T_{N}^{*} \Omega^{\prime}=\left.\operatorname{Char} \tilde{\mathbf{L}}\right|_{N}
$$

If $\left.\gamma \in \mathrm{Char} \mathbf{L}\right|_{N}$, (2.2) gives

$$
\mathcal{O}\left(H_{T N}, \gamma\right)=\mathcal{O}\left(H_{\mathrm{Re} \tilde{\mathbf{L}}}, \gamma\right) \text {. }
$$

If $u$ is a solution for $(\Omega, \mathbf{L})$ then $u^{\prime}=\left.u\right|_{\Omega^{\prime}}$ is a solution for $\left(\Omega^{\prime}, \tilde{\mathbf{L}}\right)$, and hence the singularities of $u^{\prime}$ propagate along $\Theta\left(H_{\operatorname{Re} \tilde{\mathbf{L}}}, \gamma\right)$.

Through each point $\left(x_{0}, y_{0}\right) \in \Omega^{\prime}$, the maximally real manifold

$$
\left\{(x, y) \in \Omega: y=y_{0}\right\} \subseteq \Omega^{\prime} .
$$

Since microlocal hypoanalyticity of a solution in $\Omega$ is determined by its trace on maximally real submanifolds, it follows that the singularities of $u$ propagate along $\mathcal{O}\left(H_{\operatorname{Re} \tilde{\mathrm{L}}}, \gamma\right)$ and hence along $\mathcal{O}\left(H_{T N}, \gamma\right)$. Moreover,

$$
\left.\operatorname{Char} \mathbf{L}\right|_{N}=\bigcup_{\left.\gamma \in \operatorname{Char} \mathbf{L}\right|_{N}} \mathcal{O}\left(H_{\mathrm{Re} \tilde{\mathbf{L}}}, \gamma\right) \text {. }
$$

The latter yields both Corollary 2.1 and Corollary 2.2 in this case.

Case 3: $\Omega$ is not a $C R$ structure $(n>r)$ and $N$ is elliptic, $\operatorname{dim} N=2 m^{\prime}+s$. We may assume that the restrictions of $Z_{1}, \ldots, Z_{m^{\prime}}$ generate the structure bundle on $N$. As in Case 2, we can get coordinates $x, y$ and a hypoanalytic chart such that

$$
\begin{aligned}
& Z_{j}=x_{j}+\sqrt{-1} y_{j} \text { for } 1 \leq j \leq r \text { and } \\
& Z_{k}=x_{k}+\sqrt{-1} \Phi_{k}(x, y) \text { for } r+1 \leq k \leq m
\end{aligned}
$$

and on $N$,

$$
x_{k}=0, k=m^{\prime}+1, \ldots, m ; \quad y_{l}=0, l=m^{\prime}+1, \ldots, r .
$$

Moreover, $\Phi_{\left.k\right|_{N}}=0$ for $k=r+1, \ldots, m$.

Unlike Case 2, (2.3) may not be all the defining functions for $N$. However, by applying the implicit function theorem as in [5] we may assume that $N$ is given by

$$
x_{k}=0, k=m^{\prime}+1, \ldots, m ; \quad y_{l}=0, l=m^{\prime}+1, \ldots, n-s .
$$

We adopt here the following notation:

$$
\begin{gathered}
x^{\prime}=\left(x_{1}, \ldots, x_{m^{\prime}}\right), y^{\prime}=\left(y_{1}, \ldots, y_{m^{\prime}}\right), z^{\prime}=x^{\prime}+\sqrt{-1} y^{\prime} ; \\
x^{\prime \prime}=\left(x_{m^{\prime}+1}, \ldots, x_{m}\right), y^{\prime \prime}=\left(y_{m^{\prime}+1}, \ldots, y_{n-s}\right) ; \\
y^{*}=\left(y_{n-s+1}, \ldots, y_{n}\right) .
\end{gathered}
$$

We contract $\Omega$ about 0 and assume that

$$
\Omega=\Delta^{\prime} \times V^{\prime \prime} \times W^{\prime \prime} \times W^{*},
$$

where 


$$
\Delta^{\prime}=\left\{z \in C^{m^{\prime}}:\left|z_{j}\right|<\delta, j=1, \ldots, m^{\prime}\right\}
$$

and $V^{\prime \prime}, W^{\prime \prime}, W^{*}$ are open balls centered at the origin in the spaces of $x^{\prime \prime}, y^{\prime \prime}$, and $y^{*}$, respectively. Then

$$
\begin{gathered}
N=\Delta^{\prime} \times\{0\} \times\{0\} \times W^{*} \text { and } \\
\Phi_{k}\left(x^{\prime}, y^{\prime}, 0,0, y^{*}\right)=0, \quad d \Phi_{k}(0)=0 \forall k .
\end{gathered}
$$

In these coordinates $\mathbf{L}$ is spanned by a basis of the form

$$
\begin{aligned}
& L_{j}=\frac{\partial}{\partial \bar{z}_{j}}+\sum_{k=r+1}^{m} a_{j}^{k}(x, y) \frac{\partial}{\partial x_{k}} \quad \text { for } 1 \leq j \leq r ; \\
& L_{j}=\frac{\partial}{\partial y_{j}}+\sum_{k=r+1}^{m} a_{j}^{k}(x, y) \frac{\partial}{\partial x_{k}} \text { for } r+1 \leq j \leq n .
\end{aligned}
$$

Let $\Omega^{\prime}=\Delta^{\prime} \times V^{\prime \prime} \times\{0\} \times W^{*}$. The vector fields $L_{1}, \ldots, L_{m^{\prime}}$ together with $L_{n-s+1}, \ldots, L_{n}$ are all tangent to $\Omega^{\prime}$. Let $\mathbf{L}^{\prime}$ denote the bundle on $\Omega^{\prime}$ generated by these vector fields. The restrictions of $Z_{1}, \ldots, Z_{m}$ to $\Omega^{\prime}$ generate the orthogonal of $\mathbf{L}^{\prime}$ in $T^{*} \Omega^{\prime}$. We now claim that $N=\Delta^{\prime} \times\{0\} \times\{0\} \times W^{*}$ is an orbit of $\operatorname{Re} \mathbf{L}^{\prime}$. To see this, it suffices to show that in (2.6)

and

$$
a_{j}^{k}(x, y)=0 \text { on } N \text { for } 1 \leq j \leq m^{\prime} \text { and } r+1 \leq k \leq m
$$

$$
a_{j}^{k}(x, y)=0 \text { on } N \text { for } n-s+1 \leq j \leq n \text { and } r+1 \leq k \leq m .
$$

Fix $j \in\left\{1, \ldots, m^{\prime}\right\}$. The equations

$$
L_{j}\left(x_{k}+\sqrt{-1} \phi_{k}(x, y)\right)=0 \text { for } r+1 \leq k \leq m
$$

lead to the system

$$
\sqrt{-1} \frac{\partial \phi_{k}}{\partial \bar{z}_{j}}+a_{j}^{k}+\sqrt{-1} \sum_{t=r+1}^{m} a_{j}^{t} \frac{\partial \phi_{k}}{\partial x_{t}}=0 \text { for } r+1 \leq k \leq m .
$$

By (2.5), since $\left.\phi_{k}\right|_{N}=0$ and $\partial / \partial \bar{z}_{j}$ is tangent to $N$, the functions $\partial \phi_{k} / \partial \bar{z}_{j}$ vanish on $N$. Therefore, on $N,(2.7)$ becomes

$$
a_{j}^{k}+\sqrt{-1} \sum_{t=r+1}^{m} a_{j}^{t} \frac{\partial \phi_{k}}{\partial x_{t}}=0 \text { for } r+1 \leq k \leq m .
$$

Since $d \phi_{k}(0)=0 \forall k$, it follows that in a neighborhood of 0 , and hence without loss of generality on all of $N$, we have

$$
a_{j}^{k}=0 \text { for } r+1 \leq k \leq m \text { and } 1 \leq j \leq m^{\prime} .
$$

Similar reasoning gives

$$
a_{j}^{k}=0 \text { on } N \text { for } r+1 \leq k \leq m \text { and } n-s+1 \leq j \leq n \text {. }
$$

From (2.9) and (2.10), we conclude that

$$
\left.\operatorname{Re} \mathbf{L}^{\prime}\right|_{N}=T N \text { and hence } N=\mathcal{O}\left(\operatorname{Re} \mathbf{L}^{\prime}, 0\right) .
$$


The latter permits us to apply Theorem 1.1 to obtain: if $h \in D^{\prime}\left(\Omega^{\prime}\right), \mathbf{L}^{\prime} h=0$ and $\gamma \in T_{N}^{*} \Omega^{\prime}$ then

$$
\gamma \in W F_{h a} h \Leftrightarrow \mathcal{O}\left(H_{\mathrm{Re} \mathbf{L}^{\prime}}, \gamma\right) \subseteq W F_{h a} h .
$$

We now note that the canonical map

$$
\pi:\left.T^{*} \Omega\right|_{\Omega^{\prime}} \rightarrow T^{*} \Omega^{\prime}
$$

is an injection of Char $\left.\mathbf{L}\right|_{N}$ into Char $\left.\mathbf{L}^{\prime}\right|_{N}$, and that if $\mathbf{L} u=0$ in $\Omega$ then $\mathbf{L}^{\prime} u^{\prime}=0$ in $\Omega^{\prime}$, where $u^{\prime}=\left.u\right|_{\Omega^{\prime}}$. Moreover, $\Omega^{\prime}$ contains a maximally real submanifold through each point of $N$. It follows that if $\left.\gamma \in \operatorname{Char} \mathbf{L}\right|_{N}$,

$$
\gamma \in W F_{h a} u \Leftrightarrow \pi(\gamma) \in W F_{h a} u^{\prime} .
$$

From the latter, (2.11), and (2.12) we conclude that

$$
\left.\gamma \in W F_{h a} u \Leftrightarrow \mathcal{O}\left(H_{T N}, \gamma\right) \cap \operatorname{Char} \mathbf{L}\right|_{N} \subseteq W F_{h a} u .
$$

To get Corollary 2.2, suppose $u$ is hypoanalytic at $p \in N$. Then $u^{\prime}$ is also hypoanalytic there, and hence by (2.11) and (2.12) $u^{\prime}$ is hypoanalytic at every point of $N$. But then by (2.13) $u$ is hypoanalytic at every point of $N$.

Corollary 2.3 (Hanges-Sjöstrand [4]). Suppose that the structure $(\Omega, \mathbf{L})$ is real analytic, and assume that

$$
\mathcal{O}\left(H_{\mathrm{Re} \mathbf{L}}, \gamma\right) \subseteq \text { Char } \mathbf{L} .
$$

If $u$ is a solution, then

$$
\gamma \in W F_{a} u \Leftrightarrow \mathcal{O}\left(H_{\mathrm{Re} \mathbf{L}}, \gamma\right) \subseteq W F_{a} u .
$$

Here $W F_{a} u$ denotes the analytic wavefront set of $u$ as defined in [9].

Proof. This follows from Theorem 1.2. Indeed, for solutions of a real analytic structure, the notion of microlocal analyticity coincides with that of microlocal hypoanalyticity, as demonstrated in [1] and [6].

\section{A Lemma on Microlocal Hypoanalyticity}

Let $(\Omega, Z)$ be as in Section 1 with the $Z_{j}$ given by (1.1). We first briefly recall Sato's version of microlocal hypoanalyticity (see [1] for details).

We assume $\Omega=U \times W$, where $U$ is an open ball about 0 in $x$-space in $\mathbf{R}^{m}$ and $W$ is one about 0 in $y$-space $\mathbf{R}^{n}$. Microlocal hypoanalyticity is defined for distributions in the maximally real manifold $U$.

In what follows $\Gamma$ is a nonempty, acute and open cone in $R_{m} \backslash\{0\}$. For $A$ an open subset of $U$, we shall use the notation

$$
N_{\delta}(A, \Gamma)=\left\{Z(x)+\sqrt{-1} Z_{x}(x) v: x \in A, v \in \Gamma,|v|<\delta\right\} .
$$

In this section, $Z(x)=Z(x, 0)$. 
Definition 3.1. We denote by $B_{\delta}(A, \Gamma)$ the space of holomorphic functions $f$ in $N_{\delta}(A, \Gamma)$ satisfying the condition: To every compact subset $K$ of $N_{\delta}(A, \Gamma)$ there exists an integer $k \geq 0$ and a constant $c>0$ such that

$$
|f(z)| \leq c(\operatorname{dist}[z, Z(A)])^{-k} \text { for all } z \text { in } K .
$$

In [1] it is shown that if $A$ is small enough and $f \in B_{\delta}(A, \Gamma)$ then for every $\psi \in C_{c}^{\infty}(A)$,

$$
\lim _{t \rightarrow+0} \int_{A} f\left(Z(x)+\sqrt{-1} Z_{x}(x) t v\right) \psi(x) d Z(x)
$$

exists and is independent of $v \in \Gamma$. The notation $b f$ will be used for this limit distribution.

Definition 3.2. Let $u \in D^{\prime}(U)$ and $(x, \xi) \in U \times\left(R_{m} \backslash\{0\}\right) . u$ is said to be hypoanalytic at $(x, \xi)$ if there is an open neighborhood $A \subseteq U$ of $x, \delta>0$, and a finite collection of nonempty acute open cones $\Gamma_{k}$ in $R_{m} \backslash\{0\}(k=1, \ldots, r)$ such that the following hold:

(a) for every $k$ and every $v \in \Gamma_{k}, \xi \cdot v<0$;

(b) for each $k$ there is an $f_{k} \in B_{\delta}\left(A, \Gamma_{k}\right)$ such that $u=b f_{1}+\cdots+b f_{r}$ in $A$.

We remark that the preceding definition of microlocal hypoanalyticity does not depend on the chart $(U, Z)$.

Definition 3.3. Let $u \in D^{\prime}(\dot{U})$. The hypoanalytic wavefront set of the distribution $u$ is denoted by $W F_{h a} u$ and is defined by

$$
W F_{h a} u=\left\{(x, \xi) \in U \times\left(R_{m} \backslash\{0\}\right): u \text { is not hypoanalytic at }(x, \xi)\right\} .
$$

We next recall the Fourier transform criterion of hypoanalyticity. First, we contract the neighborhood $U$ about 0 sufficiently so that the mapping

$$
Z=\left(Z_{1}, \ldots, Z_{m}\right): U \rightarrow C^{m}
$$

is a diffeomorphism onto $Z(U)$.

In (1.1) the $Z_{j}$ were chosen so that the Jacobian $Z_{x}(0)$ is the identity matrix. We now select the $Z_{j}$ so that all the derivatives of the $\phi_{j}$ up to order 2 vanish at zero. Indeed, it suffices to replace the $Z_{j}$ of (1.1) by

$$
Z_{j}-\frac{\sqrt{-1}}{2} \sum_{k} \sum_{t} \frac{\partial^{2} \phi_{j}}{\partial x_{k} \partial x_{l}}(0) Z_{k} Z_{l}
$$

(In the notation of $(1.1)$, here $\phi_{j}=y_{j}$ when $1 \leq j \leq r$.) We will use $Z_{x}^{*}$ to denote the transpose of the inverse of the matrix $Z_{x}$.

In what follows, $\mathbf{C}_{m}$ and $R_{m}$ will denote respectively the duals of complex $m$ space $\mathbf{C}^{m}$ and real space $R^{m}$.

For $\zeta$ in $\mathbf{C}^{m}$ (or $\mathbf{C}^{m}$ ), $\zeta^{2}$ will denote the sum $\sum_{j=1}^{m} \zeta_{j}^{2}$. For $\zeta$ in $\mathbf{C}_{m}$ with $|\operatorname{Im} \zeta|<|\operatorname{Re} \zeta|$, the notation $\langle\zeta\rangle$ will be used for the holomorphic branch of the square root of $\xi^{2}$ that agrees with $|\xi|$ when $\xi$ is in $R_{m}$. 
Since the first and second derivatives of all the $\phi_{j}$ are zero at the origin, after contracting $U$ if necessary we can find a number $K(0<K<1)$ such that for all $x, y \in U$ and for all $\xi \in R_{m}$,

$$
\left|\operatorname{Im} Z_{x}^{*}(x) \xi\right|<K\left|\operatorname{Re} Z_{x}^{*}(x) \xi\right|
$$

and

$$
\begin{aligned}
\operatorname{Re}\left\{\sqrt{-1} Z_{x}^{*}(x) \xi \cdot(Z(x)-Z(y))-\left\langle Z_{x}^{*}(x) \xi\right\rangle\right. & \left.(Z(x)-Z(y))^{2}\right\} \\
& \leq-K|\xi||Z(x)-Z(y)|^{2} .
\end{aligned}
$$

Let $u \in \mathcal{E}^{\prime}(U)$. The integral

$$
F(u, z, \zeta)=\int_{U} \exp \left(\sqrt{-1} \zeta(z-Z(y))-\langle\zeta\rangle(z-Z(y))^{2}\right) u(y) d Z(y)
$$

is said to be the Fourier-Bros-Iagolnitzer (in short, FBI) transform of $u$ (see [9] and [1]). Here $z \in C^{m}$ and $\zeta \in C_{m}$ with $|\operatorname{Im} \zeta|<|\operatorname{Re} \zeta|$.

In [1] the authors showed the equivalence between exponential decay in the FBI transform of $u$ and microlocal hypoanalyticity as defined in this section. They established the following theorem.

THEOREM 3.1 [1]. The following two properties are equivalent:

(i) $u$ is hypoanalytic at $\left(0, \xi^{0}\right)$ for $\xi^{0} \neq 0$.

(ii) There are open neighborhoods $V$ of 0 in $C^{m}$, a conic open neighborhood $\mathfrak{C}_{0}$ of $\xi_{0}$ in $C_{m}$, and constants $c, r>0$ such that $|F(u, z, \zeta)| \leq$ $c \exp (-r|\zeta|)$ for all $z$ in $V$ and for all $\zeta$ in $\mathfrak{C}_{0}$.

We emphasize here that Theorem 3.1 is a statement about the central point 0 , and indeed in [1] the vanishing of the derivatives of the $\phi_{j}$ at 0 was used in the proof. For the proof of Theorem 1.1 we will need the following lemma. We assume that the neighborhood $U$ has been contracted so that (3.1) holds.

LEMma 3.1. There is a neighborhood $U^{\prime}$ of $0, U^{\prime} \subseteq U$, such that for any $u \in \mathcal{E}^{\prime}\left(U^{\prime}\right)$, the following properties are equivalent:

(i) $u$ is hypoanalytic at $\left(x_{0}, \xi_{0}\right) \in U^{\prime} \times R_{m}$ for $\xi^{0} \neq 0$.

(ii) There is an open neighborhood $V$ of $Z\left(x_{0}\right)$ in $C^{m}$, a conic open neighborhood $\mathrm{e}_{0}$ of $Z_{x}^{*}\left(x_{0}\right) \xi^{0}$ in $C_{m}$, and constants $c, r>0$ such that

$$
|F(u, z, \zeta)| \leq c \exp (-r|\zeta|)
$$

for all $z$ in $V$ and for all $\zeta$ in $\mathfrak{C}_{0}$.

Proof. Suppose (i) holds. According to the definition, it suffices to prove the result when $u$ is the boundary value of a holomorphic function $f$ of tempered growth defined in a set of the form

$$
\left\{Z(x)+\sqrt{-1} Z_{x}(x) v: x+\sqrt{-1} v \in(W+\sqrt{-1} \Gamma),|v|<\delta_{0}\right\},
$$


where $W \subseteq U$ is an open neighborhood of $x_{0}, \delta_{0}$ is a positive number, and $\Gamma$ is an acute open cone in $R_{m} \backslash\{0\}$ such that for every $v \in \Gamma, \xi^{0} \cdot v<0$. Thus, for $\phi \in C_{c}^{\infty}(W)$,

$$
\langle u, \phi\rangle=\lim _{t \rightarrow+0} \int f\left(Z(x)+t \sqrt{-1} Z_{x}(x) v\right) \phi(x) d Z(x) \text { for } v \in \Gamma .
$$

After contracting $\Gamma$ if necessary, we may assume that there is a number $c_{0}>0$ such that $\xi^{0} \cdot v \leq-c_{0}\left|v \| \xi^{0}\right|$ whenever $v$ is in $\Gamma$. We shall need the following lemma.

LEMma 3.1'. Suppose $u \in \mathcal{E}^{\prime}(U)$ vanishes in an open neighborhood of $x_{0} \in U$. Then there is an open neighborhood $V$ of $Z\left(x_{0}\right)$ in $C^{m}$, a conic neighborhood $\mathcal{C}$ of $\left\{Z_{x}^{*}\left(x_{0}\right) \xi: \xi \in R_{m} \backslash\{0\}\right\}$ in $C_{m}$, and constants $c, r$ such that $|F(u, z, \zeta)| \leq$ $c e^{-r|\zeta|}$ for all $z$ in $V$ and for all $\zeta$ in $\mathcal{C}$.

Proof. For $z \in C^{m}, \zeta \in C_{m}$, and $|\operatorname{Im} \zeta|<|\operatorname{Re} \zeta|$, we consider the FBI

$$
F(u, z, \zeta)=\int \exp \left(\sqrt{-1} \zeta \cdot(z-Z(y))-\langle\zeta\rangle(z-Z(y))^{2}\right) u(y) d Z(y) .
$$

Let

$$
Q(z, \zeta, y)=\operatorname{Re}\left\{\sqrt{-1} \frac{\zeta}{|\zeta|} \cdot(z-Z(y))-\frac{\langle\zeta\rangle}{|\zeta|}(z-Z(y))^{2}\right\}
$$

We first freeze $z$ to $Z\left(x_{0}\right)$ and $\zeta$ to $Z_{x}^{*}\left(x_{0}\right) \cdot \xi^{0}$ for some $\xi^{0} \in R_{m},\left|\xi^{0}\right|=1$ :

$$
\begin{aligned}
& Q\left(Z\left(x_{0}\right), Z_{x}^{*}\left(x_{0}\right) \xi^{0}, y\right) \\
& \quad=\operatorname{Re}\left\{\sqrt{-1} \frac{Z_{x}^{*}\left(x_{0}\right) \xi^{0}}{\left|Z_{x}^{*}\left(x_{0}\right) \xi^{0}\right|}\left(Z\left(x_{0}\right)-Z(y)\right)-\frac{\left\langle Z_{x}^{*}\left(x_{0}\right) \xi^{0}\right\rangle}{\left|Z_{x}^{*}\left(x_{0}\right) \xi^{0}\right|}\left(Z\left(x_{0}\right)-Z(y)\right)^{2}\right\} .
\end{aligned}
$$

Condition (3.1) tells us that

$$
Q\left(Z\left(x_{0}\right), Z_{x}^{*}\left(x_{0}\right) \xi^{0}, y\right) \leq-K\left|Z\left(x_{0}\right)-Z(y)\right|^{2} .
$$

Suppose $d$ is a positive number such that $\left|y-x_{0}\right| \geq d$ whenever $y \in \operatorname{supp} u$. Then, in the support of $u, Q\left(Z\left(x_{0}\right), Z_{x}^{*}\left(x_{0}\right) \xi^{0}, y\right) \leq-K d^{2}$. By continuity, there are open neighborhoods $\tilde{V}$ of $Z\left(x_{0}\right)$ in $C^{m}$ and $\tilde{\mathrm{C}}$ of $Z_{x}^{*}\left(x_{0}\right) \xi^{0}$ in $C_{m}$ such that

$$
Q(z, \zeta, y) \leq-\frac{K d^{2}}{2} \text { for all } z \text { in } \tilde{V}, \zeta \text { in } \widetilde{\mathcal{C}} .
$$

By compactness of the unit sphere in $R_{m}$, we may assume that the open set $\widetilde{\mathcal{C}}$ contains the set $\left\{Z_{x}^{*}\left(x_{0}\right) \xi: \xi \in R_{m},|\xi|=1\right\}$. Moreover, the homogeneity of $Q$ implies that there is a conic neighborhood $\mathcal{C}$ of $\left\{Z_{x}^{*}\left(x_{0}\right) \xi: \xi \in R_{m} \backslash\{0\}\right\}$ in $C_{m}$ such that

$$
\operatorname{Re}\left\{\sqrt{-1} \zeta \cdot(z-Z(y))-\langle\zeta\rangle(z-Z(y))^{2}\right\} \leq-\frac{K d^{2}}{2}|\zeta|
$$

whenever $z$ is in $\tilde{V}$ and $\zeta$ is in $\mathcal{C}$. This gives us the required decay of $F(u, z, \zeta)$. 
Proof of Lemma 3.1. Let $g \in C_{c}^{\infty}(W)$ with $g \equiv 1$ near $x_{0}$. Since $(1-g) u$ vanishes near $x_{0}$, by Lemma $3.1^{\prime}$ we know that $F((1-g) u, z, \zeta)$ decays exponentially in the sets of interest. Therefore it suffices to show a similar decay for $F(g u, z, \zeta)$. Let $\chi \in C_{c}^{\infty}(W)$ with $\chi \equiv 1$ near $x_{0}$ and supp $\chi \subseteq\{x: g(x) \equiv 1\}$. Fix $v \in \Gamma$ with $|v|=1$. When $s$ is a suitably small positive number, we can deform the contour of integration in $F(g u, z, \zeta)$ under the mapping

Thus

$$
Z(y) \rightarrow \tilde{Z}(y)=Z(y)+\sqrt{-1} s Z_{y}(y) \chi(y) v .
$$

$$
\begin{aligned}
& F(g u, z, \zeta) \\
& \quad=\int_{U} \exp \left(\sqrt{-1} \zeta \cdot(z-\tilde{Z}(y))-\langle\zeta\rangle(z-\tilde{Z}(y))^{2}\right) f(\tilde{Z}(y)) \cdot g(y) d \tilde{Z}(y) .
\end{aligned}
$$

We focus on the quantity

$$
Q(z, \zeta, y, s)=\operatorname{Re}\left\{\sqrt{-1} \frac{\zeta}{|\zeta|} \cdot(z-\tilde{Z}(y))-\frac{\langle\zeta\rangle}{|\zeta|} \cdot(z-\tilde{Z}(y))^{2}\right\}
$$

and write it as $Q=Q_{1}+Q_{2}$, where

and

$$
Q_{1}(z, \zeta, y)=\operatorname{Re}\left\{\sqrt{-1} \frac{\zeta}{|\zeta|} \cdot(z-Z(y))-\frac{\langle\zeta\rangle}{|\zeta|} \cdot(z-Z(y))^{2}\right\}
$$

$$
\begin{aligned}
& Q_{2}(z, \zeta, y, s)=\operatorname{Re}\left\{\frac{\zeta}{|\zeta|} \cdot\left(s Z_{y}(y) \chi(y) v\right)\right. \\
&+\frac{\langle\zeta\rangle}{|\zeta|}\left[2 \sqrt{-1} s(z-Z(y)) \cdot\left(\chi(y) Z_{y}(y) v\right)\right. \\
&\left.\left.+s^{2}\left|\chi(y) Z_{y}(y) v\right|^{2}\right]\right\} .
\end{aligned}
$$

We first consider these quantities when $z=Z\left(x_{0}\right), \zeta=Z_{x}^{*}\left(x_{0}\right) \cdot \xi^{0}$, and $y$ varies in the support of $g$. From (3.1) we have

$$
Q_{1}\left(Z\left(x_{0}\right), Z_{x}^{*}\left(x_{0}\right) \xi^{0}, y, s\right) \leq-K\left|Z\left(x_{0}\right)-Z(y)\right|^{2} .
$$

To estimate $Q_{2}\left(Z\left(x_{0}\right), Z_{x}^{*}\left(x_{0}\right) \xi^{0}, y, s\right)$, we note that for $s$ sufficiently small, say $0<s \leq s_{0}$,

$$
\begin{aligned}
Q_{2}\left(Z\left(x_{0}\right), Z^{*}\left(x_{0}\right) \xi^{0}, x_{0}, s\right) & =\operatorname{Re}\left\{\frac{s\left(\xi^{0} \cdot v\right)}{\left|Z_{x}^{*}\left(x_{0}\right) \xi^{0}\right|}+\frac{\left\langle Z_{x}^{*}\left(x_{0}\right) \xi^{0}\right\rangle}{\left|Z_{x}^{*}\left(x_{0}\right) \xi^{0}\right|} s^{2}\left|Z_{x}\left(x_{0}\right) v\right|^{2}\right\} \\
& \leq-s c_{0} / 4 .
\end{aligned}
$$

Therefore, by continuity we can find a number $d>0$ satisfying

$$
\left|y-x_{0}\right| \leq d \Rightarrow Q_{2}\left(Z\left(x_{0}\right), Z_{x}^{*}\left(x_{0}\right) \xi^{0}, y, s\right) \leq-s c_{0} / 4 .
$$

We may assume that $\chi(y)=1$ whenever $\left|y-x_{0}\right| \leq d$. On the other hand, for each $y$,

$$
Q_{2}\left(Z\left(x_{0}\right), Z_{x}^{*}\left(x_{0}\right) \xi^{0}, y, s\right) \leq 4 s \chi(y)\left(\left|Z\left(x_{0}\right)-Z(y)\right|+s\right) .
$$


Hence, when $\left|y-x_{0}\right| \leq d$,

$$
Q\left(Z\left(x_{0}\right), Z_{x}^{*}\left(x_{0}\right) \xi^{0}, y, s\right) \leq-K\left|Z\left(x_{0}\right)-Z(y)\right|^{2}-s c_{0} / 2,
$$

while if $\left|y-x_{0}\right| \geq d$ then $\left|Z(y)-Z\left(x_{0}\right)\right| \geq d$, so that

$Q\left(Z\left(x_{0}\right), Z_{x}^{*}\left(x_{0}\right) \xi^{0}, y, s\right) \leq-K d\left|Z\left(x_{0}\right)-Z(y)\right|+4 s \chi(y)\left(\left|Z\left(x_{0}\right)-Z(y)\right|+s\right)$.

Therefore, by choosing $s$ small in comparison with $d$, we get a positive number $\delta$ such that

$$
Q\left(Z\left(x_{0}\right), Z_{x}^{*}\left(x_{0}\right) \xi^{0}, y, s\right) \leq-\delta \quad \text { when } y \in \operatorname{supp} g .
$$

By continuity, there are open neighborhoods $\tilde{V}$ of $Z\left(x_{0}\right)$ in $C^{m}$ and $\widetilde{\mathcal{C}}$ of $Z_{x}^{*}\left(x_{0}\right) \xi^{0}$ in $C_{m}$ such that $Q(z, \zeta, y, s) \leq-\delta / 2$ for all $z \in \tilde{V}, \zeta \in \widetilde{\mathcal{C}}$ and for all $y \in \operatorname{supp} g$. Now $Q$ is positive homogeneous of degree 0 in $\zeta$. Therefore, there is an open conic neighborhood $\mathrm{e}$ of $Z_{x}^{*}\left(x_{0}\right) \xi^{0}$ in $C_{m}$ such that

$$
\operatorname{Re}\left\{\sqrt{-1} \zeta \cdot(z-\tilde{Z}(y))-\langle\zeta\rangle(z-\tilde{Z}(y))^{2}\right\} \leq-\frac{\delta}{2}|\zeta|
$$

whenever $z$ is in $\tilde{V}$ and $\zeta$ in $\mathcal{C}$. From this we get the required decay of $F(u, z, \zeta)$.

That (ii) implies (i) can be seen by a slight modification of the arguments used in [1] and [9] to prove Theorem 3.1. We will therefore only give a short outline. The main idea is to use the inversion of the FBI transform. For $M$ a compact neighborhood of 0 in $U$, define the set $T_{M}$ by

$$
T_{M}=\left\{(z, \zeta): z=Z(x), \zeta=Z_{x}^{*}(x) \xi \text { for some }(x, \xi) \in M \times R_{m} \backslash\{0\}\right\} .
$$

$\Delta(z, \zeta)$ will denote the Jacobian $\operatorname{det}(\partial \theta / \partial \zeta)$, where $\theta=\zeta+\sqrt{-1}\langle\zeta\rangle z$. For $\delta>0$ and $h \in \mathcal{E}^{\prime}(U)$, define the holomorphic function

$$
\begin{array}{r}
h_{M}^{\delta}(z)=\left(4 \pi^{3}\right)^{-m / 2} \int_{T_{M}} \int \exp \left(\sqrt{-1}(z-w) \cdot \zeta-\langle\zeta\rangle(z-w)^{2}-\delta\langle\zeta\rangle^{2}\right) \\
\cdot F(h, w, \zeta)\langle\zeta\rangle^{m / 2} \Delta(z-w, \zeta) d w d \zeta
\end{array}
$$

We will need the following lemma from [12].

Lemma 3.1". Let $M$ be as above. There exist two open sets $U_{0}$ and $U_{1}$ containing 0 , with $U_{0} \subseteq U_{1} \subseteq U$, such that if $h \in \mathcal{E}^{\prime}\left(U_{1}\right)$ then $h_{M}^{\delta} Z \rightarrow h+f \circ Z$ in $D^{\prime}\left(U_{0}\right)$, where $f$ is holomorphic in an open set in $C^{m}$ containing $Z\left(U_{0}\right)$.

Fix $M, U_{0}, U_{1}$ as in the lemma, and let $U^{\prime}=M \cap U_{0}$. Let $x_{0}$ be in $U^{\prime}$ and suppose that (ii) holds for $x_{0}$ and $u \in \mathcal{E}^{\prime}\left(U^{\prime}\right)$. Define $\Gamma_{0}=\left(V \times \mathcal{C}_{0}\right) \cap T_{M}$, where $V$ and $\mathcal{C}_{0}$ are the neighborhoods of $Z\left(x_{0}\right)$ and $Z_{x}^{*}\left(x_{0}\right) \xi^{0}$ (respectively) satisfying (ii).

Let $\Gamma_{1}, \ldots, \Gamma_{k}$ be conically compact sets such that

(i) $T_{M}=\bigcup_{j=0}^{k} \Gamma_{j}$;

(ii) measure $\left(\Gamma_{i} \cap \Gamma_{j}\right)=0$ when $i \neq j$; and

(iii) for each $j=1, \ldots, k$ there is a convex open subset $\Gamma_{j}^{\prime} \subseteq U_{0} \times \mathbf{R}^{m}$ whose base contains $x_{0}$ and which satisfies the following property: 


$$
v \cdot \xi^{0}<0, \quad v \cdot \xi \geq d|v||\xi| \quad(d>0)
$$

if $(x, v) \in \Gamma_{j}^{\prime}$ and $\left(Z(x), Z_{x}^{*}(x) \xi\right) \in \Gamma_{j}$ for some $x$.

Let $u_{j}^{\delta}$ denote the integral (3.2) in which the integration is carried out over $\Gamma_{j}$. For $u_{0}^{\delta}(z)$ we use (3.1) and (ii) to estimate the integrand as follows: If $z=Z\left(x_{0}\right)$ and $(w, \zeta)=\left(Z(x), Z_{x}^{*}(x) \xi\right) \in \Gamma_{0}$, then

$$
\begin{aligned}
\left|\exp \left(\sqrt{-1}(z-w) \cdot \zeta-\langle\zeta\rangle(z-w)^{2}\right) F(u, w, \zeta)\langle\zeta\rangle^{m / 2} \Delta(z-w, \zeta)\right| \\
\leq \mathrm{const}|\zeta|^{m / 2} \exp \left(-K|\xi|\left|Z\left(x_{0}\right)-Z(x)\right|^{2}-r\left|Z_{x}^{*}(x) \xi\right|\right) .
\end{aligned}
$$

Recall also that $Z_{x}^{*}(x)$ is very close to the identity matrix. Hence when $\delta \rightarrow 0$, $u_{0}^{\delta}$ converges uniformly to a holomorphic function in a neighborhood of $z=Z\left(x_{0}\right)$. Fix $j \geq 1$. We will show that $u_{j}^{\delta}(z)$ converges uniformly on the set $\left\{z: z=Z(x)+\sqrt{-1} Z_{x}(x) v,(x, v) \in \Gamma_{j}^{\prime}\right\}$ when $v$ is small.

Let $(x, v) \in \Gamma_{j}^{\prime}$. If $z=Z(x)+\sqrt{-1} Z_{x}(x) v$ and $(w, \zeta)=\left(Z\left(x^{\prime}\right), Z_{x}^{*}(x) \xi\right) \in$ $\Gamma_{j}$, then using (3.1) and (3.3) one gets the estimate (see [12])

$$
\begin{aligned}
\left|\exp \left(\sqrt{-1}(z-w) \cdot \zeta-\langle\zeta\rangle(z-w)^{2}\right) F(u, w, \zeta)\langle\zeta\rangle^{m / 2} \Delta(z-w, \zeta)\right| \\
\leq \operatorname{const}(1+|\zeta|)^{N-m-1} \exp \left(-\tilde{c}\left(|v|+|z-w|^{2}\right)|\zeta|\right)
\end{aligned}
$$

Here $N$ is the order of the distribution $u$.

This estimate implies that as $\delta \rightarrow 0$, the function $|v|^{N} u_{j}^{\delta}\left(Z(x)+\sqrt{-1} Z_{x}(x) v\right)$ converges uniformly. This proves that the limit of $u_{j}^{\delta}(Z(x))$ exists in a neighborhood of $x_{0}$ and is the boundary value of a tempered holomorphic function in a set of the form

$$
\left\{Z(x)+\sqrt{-1} Z_{x}(x) v:(x, v) \in \Gamma,|v|<\delta\right\} .
$$

It follows that $\left(x_{0}, \xi^{0}\right) \notin W F_{h a} u$.

\section{Proof of Theorems 1.1 and 1.2}

In this section $(\Omega, Z)$ is as in Section 1 with $Z=\left(Z_{1}, \ldots, Z_{m}\right)$ satisfying (1.1). To exploit the theorems of Trépreau, we will begin by first associating a $C R$ structure to $\Omega^{\prime}=\Omega \times T$, where $T$ is a neighborhood of 0 in $\mathbf{R}^{n-r}$. We will use the variable $t=\left(t_{r+1}, \ldots, t_{n}\right)$ for points in $T$. The structure bundle in $\Omega^{\prime}$ is generated by

$$
\begin{aligned}
& Z_{j}=x_{j}+\sqrt{-1} y_{j} \text { for } 1 \leq j \leq r ; \\
& Z_{k}=x_{k}+\sqrt{-1} \phi_{k}(x, y) \text { for } r+1 \leq k \leq m \text {; } \\
& W_{k}=t_{r+k}+\sqrt{-1} y_{r-k} \quad \text { for } 1 \leq k \leq n-r \text {. }
\end{aligned}
$$

We will use the notation

$$
\tilde{Z}(x, y, t)=(Z(x, y), W(t, y)) .
$$

We recall that the bundle $\mathbf{L}$ had as a basis: 


$$
\begin{aligned}
L_{j} & =\frac{\partial}{\partial \bar{z}_{j}}+\sum_{k=r+1}^{m} b_{j k} \frac{\partial}{\partial x_{k}}, \quad 1 \leq j \leq r ; \\
L_{j} & =\frac{\sqrt{-1}}{2} \frac{\partial}{\partial y_{j}}+\sum_{k=r+1}^{m} b_{j k}^{\prime} \frac{\partial}{\partial x_{k}}, \quad r+1 \leq j \leq n .
\end{aligned}
$$

The structure associated to $\Omega^{\prime}$ has a bundle $\nabla$ with basis:

$$
\begin{gathered}
V_{j}=L_{j}, \quad 1 \leq j \leq r ; \\
V_{k}=\frac{1}{2}\left(\frac{\partial}{\partial t_{k}}+\sqrt{-1} \frac{\partial}{\partial y_{k}}\right)+\sum_{j=r+1}^{m} b_{k j}^{\prime} \frac{\partial}{\partial x_{j}}, \quad r+1 \leq k \leq n .
\end{gathered}
$$

We use $\left(x^{\prime}, x^{\prime \prime}, y ; \xi^{\prime}, \xi^{\prime \prime}, \eta\right)$ for points in $T^{*} \Omega$, where $x^{\prime}=\left(x_{1}, \ldots, x_{l}\right), x^{\prime \prime}=$ $\left(x_{l+1}, \ldots, x_{m}\right), \xi^{\prime}=\left(\xi_{1}, \ldots, \xi_{l}\right)$, and $\xi^{\prime \prime}=\left(\xi_{l+1}, \ldots, \xi_{m}\right)$. Lemma 4.2 will show that if $N$ is an orbit of $\operatorname{Re} \mathbf{L}$ through 0 , coordinates $\left(x^{\prime}, x^{\prime \prime}, y\right)$ can be found for $\Omega$ in which $N$ is defined by $x^{\prime \prime}=0$.

Points in $T^{*}(\Omega \times T)$ will be denoted by $\left(x^{\prime}, x^{\prime \prime}, y, t ; \xi^{\prime}, \xi^{\prime \prime}, \eta, \tau\right)$, where $x^{\prime}, x^{\prime \prime}, \xi^{\prime}, \xi^{\prime \prime}$ are as above and $\tau=\left(\tau_{r+1}, \ldots, \tau_{n}\right)$ is dual to $t$. With this notation, we can write

$$
\begin{aligned}
T_{N}^{*} \Omega & =\left\{\left(x^{\prime}, 0, y ; 0, \xi^{\prime \prime}, 0\right):\left(x^{\prime}, 0, y\right) \in N\right\} \quad \text { and } \\
T_{N \times T}^{*}(\Omega \times T) & =\left\{\left(x^{\prime}, 0, y, t ; 0, \xi^{\prime \prime}, 0,0\right):\left(x^{\prime}, 0, y, t\right) \in N \times T\right\} .
\end{aligned}
$$

In the following lemma,

$$
\begin{aligned}
& \sigma=\left(x_{1}^{0}, \ldots, x_{l}^{0}, 0, y^{0} ; 0, \xi_{l+1}^{0}, \ldots, \xi_{m}^{0}, 0\right) \text { and } \\
& \tilde{\sigma}=\left(x_{1}^{0}, \ldots, x_{l}^{0}, 0, y^{0}, 0 ; 0, \xi_{l+1}^{0}, \ldots, \xi_{m}^{0}, 0,0\right) .
\end{aligned}
$$

Lemma 4.1. Let $u$ be a solution in $(\Omega, \mathrm{L})$ and let $\tilde{u}(x, y, t)=u(x, y)$. Then

$$
\tilde{\sigma} \notin W F_{h a} \tilde{u} \text { in }(\Omega \times T, \nabla) \Leftrightarrow \sigma \notin W F_{h a} u \text { in }(\Omega, \mathbf{L}) \text {. }
$$

Proof. Lemma 3.1 enables us to use the FBI transform. This may require the contraction of $\Omega$ about the origin. However, we note that we need only prove the propagation of Theorem 1.1 in some neighborhood independent of the solution $u$.

Since $\tilde{u}$ is a solution for $(\Omega \times T, \vartheta)$, we may use the maximally real manifold $\tilde{X}=\left\{\left(x, y^{0}, t\right)\right\}$ which contains the base projection of $\tilde{\sigma}$. For $u$ we may use $X=\left\{\left(x, y^{0}\right)\right\}$. Let $(z, w) \in C^{m} \times C^{n-r}$ and $(\zeta, \tau) \in C_{m} \times C_{n-r}$ denote variable points.

The FBI transform of $\tilde{u}\left(x, y^{0}, t\right)=u\left(x, y^{0}\right)$ in $\tilde{X}$ can be factored as

$$
F(\tilde{u}, z, w, \zeta, \tau)=I_{1}(z, \zeta, \tau) \cdot I_{2}(w, \zeta, \tau),
$$

where

$$
\begin{aligned}
& I_{1}(z, \zeta, \tau) \\
& \quad=\int \exp \left(\sqrt{-1} \zeta \cdot\left(z-Z\left(x, y_{0}\right)\right)-\langle\zeta, \tau\rangle\left(z-Z\left(x, y_{0}\right)\right)^{2}\right) u\left(x, y_{0}\right) d Z\left(x, y_{0}\right)
\end{aligned}
$$


and

$$
\begin{aligned}
& I_{2}(w, \zeta, \tau) \\
& \quad=\int_{T} \exp \left(\sqrt{-1} \tau \cdot\left(w-W\left(t, y_{0}\right)\right)-\langle\zeta, \tau\rangle\left(w-W\left(t, y_{0}\right)\right)^{2}\right) d t_{r+1} \ldots d t_{n} .
\end{aligned}
$$

If $\sigma \notin W F_{h a} u$, Lemma 3.1 tells us that there exists a neighborhood $V$ of $Z\left(x_{1}^{0}, \ldots, x_{l}^{0}, 0, y^{0}\right)$ in $C^{m}$ and a conic open neighborhood $\mathcal{C}$ of $\zeta^{0}=$ $Z_{x}^{*}\left(x_{1}^{0}, \ldots, x_{l}^{0}, 0, y^{0}\right)\left(0, \xi_{l+1}^{0}, \ldots, \xi_{m}^{0}\right)$ in $C_{m}$, together with positive constants $c_{1}$ and $c_{2}$, such that $\left|I_{1}(z, \zeta, 0)\right| \leq c_{1} \exp \left(-c_{2}|\zeta|\right)$ for $z \in V$ and $\zeta \in \mathcal{C}$. The factor $I_{2}$ satisfies an estimate of the form $\left|I_{2}(w, \zeta, \tau)\right| \leq d_{1} e^{d_{2}|\tau|}$ when $w=$ $W\left(0, y_{0}\right)$ and $(\zeta, \tau)$ satisfies $|\operatorname{Re}(\zeta, \tau)|>|\operatorname{Im}(\zeta, \tau)|$. Indeed, for such $(\zeta, \tau)$, $\operatorname{Re}\langle\zeta, \tau\rangle \geq 0$.

Therefore, for each $\epsilon>0$, there is a neighborhood $V_{\epsilon}$ of $W\left(0, y_{0}\right)$ such that $\left|I_{2}(w, \zeta, \tau)\right| \leq d_{1} e^{d_{2}|\tau|+\epsilon|\zeta|}$ whenever $w \in V_{\epsilon},|\operatorname{Re}(\zeta, \tau)|>|\operatorname{Im}(\zeta, \tau)|$.

We now recall that

$$
\tilde{Z}\left(x_{1}^{0}, \ldots, x_{l}^{0}, 0 y^{0}, 0\right)=\left(Z\left(x_{1}^{0}, \ldots, x_{l}^{0}, 0, y^{0}\right), W\left(0, y^{0}\right)\right)
$$

and

$$
\begin{aligned}
Z_{x, t}^{*}\left(x_{1}^{0}, \ldots, x_{l}^{0}, 0, y^{0}, 0\right) & \left(0, \xi_{l+1}^{0}, \ldots, \xi_{m}^{0}, 0\right) \\
& =\left(Z_{x}^{*}\left(x_{1}^{0}, \ldots, x_{l}^{0}, 0, y^{0}\right)\left(0, \xi_{l+1}^{0}, \ldots, \xi_{m}^{0}\right), 0\right)
\end{aligned}
$$

The proof of Lemma 3.1 shows that $I_{1}$ satisfies an estimate $\left|I_{1}(z, \zeta, \tau)\right| \leq$ $c_{1} \exp \left(-c_{2}|\zeta|\right)$ for $z$ in $V$ a neighborhood of 0 in $C^{m}$ and $(\zeta, \tau)$ in a conic neighborhood of $Z_{x}^{*}\left(x_{1}^{0}, \ldots, x_{l}^{0}, 0, y^{0}\right)\left(0, \xi_{l+1}^{0}, \ldots, \zeta_{m}^{0}\right)$ in $C_{m} \times C_{n-r}$, where $|\tau|<\delta|\zeta|$ for some $\delta>0$.

These estimates on $I_{1}$ and $I_{2}$ imply that there are constants $r_{1}, r_{2}>0$ such that

$$
|F(\tilde{u}, z, w, \zeta, \tau)| \leq r_{1} \exp \left(-r_{2}|(\zeta, \tau)|\right)
$$

for $(z, w)$ near $\tilde{Z}\left(x_{1}^{0}, \ldots, x_{l}^{0}, 0, y^{0}, 0\right)$ and $(\zeta, \tau)$ in a conic neighborhood of $\left(\zeta^{0}, 0\right)$ in $C_{m} \times C_{n-r}$. It follows that $\tilde{\sigma} \notin W F_{h a} \tilde{u}$.

Suppose now that $\tilde{\sigma} \notin W F_{h a} \tilde{u}$. Then Lemma 3.1 tells us that

$$
F(\tilde{u}, z, w, \zeta, \tau)=I_{1}(z, \zeta, \tau) I_{2}(w, \zeta, \tau)
$$

decays exponentially as in (4.1). In particular, $F\left(\tilde{u}, z, W\left(0, y_{0}\right), \zeta, 0\right)$ decays exponentially for $z$ near $Z\left(x_{1}^{0}, \ldots, x_{l}^{0}, 0, y^{0}\right)$ and $\zeta$ in a conic neighborhood of $\zeta^{0}$. Since the $t$ component of $\tilde{\sigma}$ is 0 , we may contract $T$ around 0 as much as we wish in the integral $I_{2}$. It follows that $I_{1}(z, \zeta, 0)$ decays exponentially for $z$ near $Z\left(x_{1}^{0}, \ldots, x_{l}^{0}, 0, y^{0}\right)$ and $\zeta$ in a conic neighborhood of $\xi^{0}$. Hence $\sigma \notin W F_{h a} u$.

LEMMA 4.2. If $N$ is an orbit through 0 for $\operatorname{Re} \mathbf{L}$ in $\Omega$, then $N \times T$ is an orbit for $\operatorname{Re} V$ in $\Omega \times T$.

Proof. We begin by first finding coordinates in $\Omega$ that flatten $N$ and leave the "form" of $\mathbf{L}$ unchanged. Since $\left.\mathbf{L}\right|_{N} \subseteq C T N$ and the fiber dimension of $\mathbf{L} \geq n$, 
$\operatorname{dim} N=n+l$ for some $0 \leq l \leq m$. Let $N$ be defined by $h_{1}=\cdots=h_{m-l}=0$. From $L_{j} h_{k}=0$ on $N$ we get

$$
\begin{aligned}
& \frac{\partial h_{k}}{\partial x_{j}}(0)=0 \quad \forall k=1, \ldots, m-l \text { and } j=1, \ldots, r ; \\
& \frac{\partial h_{k}}{\partial y_{j}}(0)=0 \quad \forall k=1, \ldots, m-l \text { and } j=1, \ldots, n .
\end{aligned}
$$

Since also the differentials of $h_{1}, \ldots, h_{m-l}$ are independent near 0 , we have

$$
\operatorname{rank}\left(\frac{\partial h_{j}}{\partial x_{k}}(0)\right)_{\substack{1 \leq j \leq m-l \\ r+1 \leq k \leq m}}=m-l .
$$

Therefore, after a possible permutation of the variables $x_{r+1}, \ldots, x_{m}$ and using the implicit function theorem, we get functions $g_{1}, \ldots, g_{m-l}$ of $x_{1}, \ldots$, $x_{l}, y_{1}, \ldots, y_{n}$ such that

$$
h_{j}(x, y)=0 \Leftrightarrow x_{l+j}=g_{j}\left(x_{1}, \ldots, x_{l}, y_{1}, \ldots, y_{n}\right), \quad 1 \leq j \leq m-l .
$$

Change coordinates in $\Omega$ by the mapping $(x, y) \mapsto(\tilde{x}, \tilde{y})$, so that

$$
\begin{gathered}
\tilde{x}_{j}= \begin{cases}x_{j}, & 1 \leq j \leq l, \\
x_{j}-g_{j-l}(x, y), & l+1 \leq j \leq m ;\end{cases} \\
\tilde{y}_{k}=y_{k} \quad \forall k=1, \ldots, n .
\end{gathered}
$$

After dropping the tildes, we have found coordinates $(x, y) \in \mathbf{R}^{m} \times \mathbf{R}^{n}$ which flatten

$$
N=\left\{(x, y) \in \Omega: x_{j}=0, j=l+1, \ldots, m\right\},
$$

and the vector fields $L_{j}$ take the form

$$
\begin{gathered}
L_{j}=\frac{\partial}{\partial \bar{z}_{j}}+\sum_{k=r+1}^{l} a_{j k} \frac{\partial}{\partial x_{k}}+\sum_{k=l+1}^{m} b_{j k} \frac{\partial}{\partial x_{k}} \quad \text { for } 1 \leq j \leq r ; \\
L_{j}=\frac{\sqrt{-1}}{2} \frac{\partial}{\partial y_{j}}+\sum_{k=r+1}^{l} \tilde{a}_{j k} \frac{\partial}{\partial x_{k}}+\sum_{k=l+1}^{m} \tilde{b}_{j k} \frac{\partial}{\partial x_{k}} \quad \text { for } r+1 \leq j \leq n,
\end{gathered}
$$

with $b_{j k}\left(x_{1}, \ldots, x_{l}, 0, y\right)=\tilde{b}_{j k}\left(x_{1}, \ldots, x_{l}, 0, y\right)=0$.

In these coordinates, $\nabla$ is still spanned by

$$
\begin{aligned}
V_{j} & =L_{j} \text { for } 1 \leq j \leq r \text { and } \\
V_{k} & =\frac{1}{2} \frac{\partial}{\partial t_{k}}+L_{k} \text { for } r+1 \leq k \leq n,
\end{aligned}
$$

where the $L_{j}$ and $L_{k}$ are as above.

Recall from Sussmann [10] that given $m_{0} \in N$ there exist $S=\left(S_{1}, \ldots, S_{q}\right) \in$ $\mathbf{R}^{q}$ for some $q, \xi=\left(X^{1}, \ldots, X^{q}\right)$ (each $\left.X^{j} \in \operatorname{Re} \mathbf{L}\right), m \in N$, and $\delta>0$ such that $\rho_{\xi, m}\left(B_{\delta}(T)\right)=\Lambda$ is a neighborhood of $m_{0}$ in the orbit $N$. Here, for $s \in \mathbf{R}^{q}$, $\rho_{\xi, m}(s)=X_{s_{q}}^{q} \ldots X_{s_{1}}^{1} m, \rho_{\xi, m}\left(S_{1}, \ldots, S_{q}\right)=X_{S_{q}}^{q} \ldots X_{S_{1}}^{\prime} m=m_{0}$, and $B_{\delta}(S)=$ $\left\{s \in \mathbf{R}^{q}:|s-S|<\delta\right\}$. Hence, if $m^{\prime} \in \Lambda, \exists s^{\prime}=\left(s_{1}^{\prime}, \ldots, s_{q}^{\prime}\right)$ such that 


$$
m^{\prime}=X_{s_{q}^{\prime}}^{q} \ldots X_{s_{1}^{\prime}}^{1} m=X_{s_{q}^{\prime}}^{q} \ldots X_{s_{1}^{\prime}}^{1} X_{-S_{1}}^{1} \ldots X_{-S_{q}}^{q} m_{0} .
$$

Let

$$
\begin{aligned}
A & =\left\{\operatorname{Re} L_{1}, \ldots, \operatorname{Re} L_{r}, \operatorname{Im} L_{1}, \ldots, \operatorname{Im} L_{n}\right\} \\
& =\left\{\operatorname{Re} V_{1}, \ldots, \operatorname{Re} V_{r}, \operatorname{Im} V_{1}, \ldots, \operatorname{Im} V_{n}\right\}
\end{aligned}
$$

and

$$
B=\left\{\operatorname{Re} V_{r+1}, \ldots, \operatorname{Re} V_{n}\right\} .
$$

Recall that $\operatorname{Re} V_{r+1}=\frac{1}{2}\left(\partial / \partial t_{r+j}\right)+\operatorname{Re} L_{r+j}$ for $j=1, \ldots, n-r$.

Suppose $\left(x^{\prime}, 0, y\right) \in N$ and $t \in T$. If $X \in A$ then

$$
X_{s}\left(x^{\prime}, 0, y, t\right)=\left(X_{s}\left(x^{\prime}, 0, y\right), t\right) .
$$

On the other hand, if $Y \in B$, say $Y=\operatorname{Re} V_{r+1}$ for definiteness, then

$$
Y_{s}\left(x^{\prime}, 0, y, t\right)=\left(\left(\operatorname{Re} L_{r+1}\right)_{s}\left(x^{\prime}, 0, y\right), t_{r+1}+\frac{s_{r+1}}{2}, t_{r+2}, \ldots, t_{n}\right) .
$$

Moreover, since $\operatorname{Re} L_{r+1}(0)=0$, we have

$$
Y_{s}(0,0, t)=\left(0,0, t_{r+1}+\frac{s_{r+1}}{2}, t_{r+2}, \ldots, t_{n}\right) .
$$

We apply the preceding conclusions to $m_{0}=0$ and contract $N$ about 0 so that $N=\Lambda$. Given $\left(x^{\prime}, 0, y\right) \in N$ and $t=\left(t_{r+1}, \ldots, t_{n}\right) \in T$, let

$$
X_{s_{1}}^{1} \ldots X_{s_{k}}^{k}(0,0)=\left(x^{\prime}, 0, y\right) \text {. }
$$

Then $\tilde{X}_{s_{1}}^{1} \ldots \tilde{X}_{s_{k}}^{k}(0,0,0)=\left(x^{\prime}, 0, y ; \tau_{r+1}, \ldots, \tau_{n}\right)$, where $\Sigma\left|\tau_{j}\right|<\delta / 2$. Here, when $X=\operatorname{Re} L_{j}, \tilde{X}=\operatorname{Re} V_{j}$. It follows that

$$
\tilde{X}_{s_{1}}^{1} \ldots \tilde{X}_{s_{k}}^{k}\left(\operatorname{Re} V_{r+1}\right)_{2 t_{r+1}-2 \tau_{r+1}} \ldots\left(\operatorname{Re} V_{n}\right)_{2 t_{n}-2 \tau_{n}}(0,0,0)=\left(x^{\prime}, 0, y, t\right) .
$$

Hence $\Lambda \times T$ is an orbit for $\operatorname{Re}\urcorner$.

Finally, we state the two results of Trépreau in [13] that we will use.

THEOREM $4.1\left[13\right.$, Thm. 10]. Suppose $M$ is a generic $C R$ manifold in $C^{n}$ and $N$ is a $C R$ submanifold of $M$, with $C R$ dimension of $N=C R$ dimension of $M$. If $u$ is a CR function on $M$ and $x^{*} \in T_{N}^{*} M \backslash\{0\}$, then

$$
x^{*} \in W F_{h a} u \Leftrightarrow \mathcal{O}\left(H_{X}, x^{*}\right) \subseteq W F_{h a} u,
$$

where $H_{X}$ is the family of Hamilton fields of the real parts of the CR vecior fields tangential to $M$.

Theorem 4.2 [13, Thm. 7]. Suppose $M$ is a generic $C R$ manifold in $C^{n}$ and $\mathscr{L} \subseteq T_{M}^{*} \mathbf{C}^{n} \backslash\{0\}$ is a minimal $C R$ manifold in $T^{*} C^{n}$. If $u$ is a $C R$ function on $M$, we have

$$
\mathfrak{L} \subseteq W F_{h a} u \Leftrightarrow \mathscr{L} \cap W F_{h a} u \neq \emptyset
$$


In Theorem 4.2, $T_{M}^{*} C^{n}$ is defined as follows: Let $T^{*} C^{n}$ denote the bundle of $(1,0)$ forms $\theta=\sum_{j=1}^{n} \zeta_{j} d z_{j}$. Then

$$
T_{M}^{*} C^{n}=\left\{(z, \theta) \in T^{*} C^{n}: z \in M,\left.\operatorname{Im} \theta\right|_{T_{z} M}=0\right\} .
$$

Proof of Theorem 1.1. Let $N=\mathcal{O}(\operatorname{Re} \mathbf{L}, 0)$. We continue to use $(z, w)=$ $\left(z_{1}, \ldots, z_{m}, w_{1}, \ldots, w_{n-r}\right)$ for a variable point in $C^{m} \times C^{n-r}$. Let $M^{\prime}$ denote the image of

$$
\tilde{Z}=\tilde{Z}(x, y, t): \Omega \times T \rightarrow C^{m} \times C^{n-r}
$$

in complex space $C^{m} \times C^{n-r} . M^{\prime}$ is a generic $C R$ manifold with a $C R$ vector bundle $=\tilde{Z}_{*}{ }^{\top}$ V. $N^{\prime}=\tilde{Z}(N \times T)$ is a submanifold of $M^{\prime}$ and by Lemma 4.2, since $\tilde{Z}_{*}{ }^{\top} \mathrm{Z}$ is tangential to $N^{\prime}$, we know that $N^{\prime}$ is a $C R$ manifold with $C R \operatorname{dim} M^{\prime}=C R \operatorname{dim} N^{\prime}$. Suppose $\mathbf{L} u=0$ in $\Omega$. If $u$ is defined on $M^{\prime}$ by $\tilde{u}(\tilde{Z}(x, y, t))=u(x, y)$, then $\tilde{u}$ is a $C R$ distribution on $M^{\prime}$. Since the Hamilton fields $H_{\operatorname{Re} \vartheta}$ are related to $H_{\operatorname{Re} \mathbf{L}}$ in the same way as $\operatorname{Re} \vee$ is related to $\operatorname{Re} \mathbf{L}$, the arguments of Lemma 4.2 lead to the following conclusion:

$$
\mathcal{O}\left(H_{\operatorname{Re} \vartheta}, \tilde{\sigma}\right)=\left\{\left(x^{\prime}, 0, y, t ; 0, \xi^{\prime \prime}, 0,0\right):\left(x^{\prime}, 0, y ; 0, \xi^{\prime \prime}, 0\right) \in \mathcal{O}\left(H_{\operatorname{Re} \mathbf{L}}, \sigma\right)\right\},
$$

where $\sigma \in T_{N}^{*} \Omega$ and $\tilde{\sigma} \in T_{N \times T}^{*}(\Omega \times T)$ as in Lemma 4.1. Theorem 1.1 now follows from Lemma 4.1 and Theorem 4.1 by transferring the problem to $M^{\prime}$, $N^{\prime}$ and $\tilde{u}$.

Proof of Theorem 1.2. As in the proof of Theorem 1.1, we transfer the problem to $M^{\prime}$ and use the lemmas of this paper. Let $\Gamma^{\prime}$ be the subset of $T^{*}(\Omega \times T)$ defined by

$$
\left.\Gamma^{\prime}=\{x, y, 0 ; \xi, \eta, 0):(x, y, \xi, \eta) \in \Gamma\right\} .
$$

Since $\Gamma \subset$ Char $\mathbf{L}$, we have $\Gamma^{\prime} \subset$ Char $\nabla$. Let $\Gamma^{\prime \prime}=$ the transfer to $M^{\prime}$ by the map $\tilde{Z}$. The condition $\Gamma^{\prime} \subseteq$ Char $\nabla$ implies that $\Gamma^{\prime \prime} \subseteq T_{M}^{*} C^{n} \backslash\{0\}$. Theorem 1.2 now follows from Lemma 4.1 and Theorem 4.2.

\section{References}

[1] M. S. Baouendi, C. H. Chang, and F. Treves, Microlocal hypo-analyticity and extension of CR functions, J. Differential Geom. 18 (1983), 331-391.

[2] M. S. Baouendi and L. P. Rothschild, Normal forms for generic manifolds and holomorphic extension of CR functions, J. Differential Geom. 25 (1987), 431-467.

[3] - Cauchy-Riemann functions on manifolds of higher codimension in complex space, Invent. Math. 101 (1990), 45-56.

[4] N. Hanges and J. Sjöstrand, Propagation of analyticity for a class of non-microcharacteristic operators, Ann. of Math. (2) 116 (1982), 559-577.

[5] N. Hanges and F. Treves, Propagation of holomorphic extendability of CR functions, Math. Ann. 263 (1983), 157-177.

[6] - On the analyticity of solutions of first-order nonlinear pde, Preprint, 1990. 
[7] M. Marson, Minimality and the extension of functions from generic manifolds, Preprint, 1989.

[8] T. Nagano, Linear differential systems with singularities and an application to transitive Lie algebras, J. Math. Soc. Japan 18 (1966), 398-404.

[9] J. Sjöstrand, Singularités analytiques micorlocales, Astérisque 95 (1982), 1-166.

[10] H. Sussmann, Orbits of families of vector fields and integrability of distributions, Trans. Amer. Math. Soc. 180 (1973), 171-188.

[11] F. Treves, Approximation and representation of functions and distributions annihilated by a system of complex vector fields, Ecole Polytechnique, Centre de Mathématiques, Palaiseau, 1981.

[12] - Hypo-analytic structures, Microlocal theory (in preparation).

[13] J. M. Trépreau, Sur la propagation des singularités dans les variétés $C R$, Preprint, 1990.

[14] A. E. Tumanov, Extending CR functions on manifolds of finite type to a wedge, Mat. Sb. (N.S.) 136 (1988), 128-139 (Russian).

Department of Mathematics

Temple University

Philadelphia, PA 19122 